\title{
AÇÕES DE PREVENÇÃO E COMBATE A HIPERTENSÃO ARTERIAL
}

\author{
Alberto Furini Vaz a, Virna Liza Pereira Chaves Hildebrand ${ }^{\text {b. }}$ \\ a Médico Clínico Geral integrante do Programa Mais Médicos para o Brasil, realizador do Projeto; Especialidade em \\ Saúde da Família, FIOCRUZ/ Mato Grosso do Sul, Ponta Porã, MS.

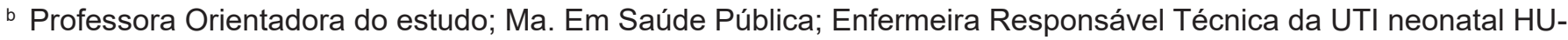 \\ MAP-UFMS; Campo Grande, MS
}

\section{RESUMO}

O objetivo do trabalho foi desenvolver ações de prevenção e combate a HAS entre os usuários da ESF rural do município de Ponta Porã/MS. Trata-se de um trabalho realizado no formato de Projeto de Intervenção (PI), com foco na realização de ações de educação em saúde. Estas ações precisaram sofrer adaptações em virtude das medidas de isolamento impostas frente a pandemia causada pelo Covid-19 e passaram da modalidade presencial para a virtual. Como resultados da proposta identificou-se que $80 \%$ dos participantes afirmaram estar consumindo menos sódio na dieta e $60 \%$ realizando atividade física regular. Houve ainda aumento das consultas médicas com o médico da família em 52\%. Como considerações finais pode-se afirmar que houve diminuição dos casos de busca por atendimento de crises hipertensivas demonstrando que o acompanhamento médico contínuo e ações de políticas de saúde num contexto multidisciplinar pode ser decisivo para garantir melhor qualidade de vida aos usuários.

Palavras-chave: Saúde da comunidade; Doenças Crônicas; Educação em saúde.

\section{ABSTRACT}

The objective of the work was to develop actions to prevent and combat SAH among users of the rural ESF in the municipality of Ponta Porã/MS. This is a work carried out in the Intervention Project (IP) format, focusing on carrying out health education actions. These actions needed to be adapted due to the isolation measures imposed in the face of the pandemic caused by Covid-19 and went from the face-to-face modality to the virtual one. As a result of the proposal, it was identified that $80 \%$ of the participants claimed to be consuming less sodium in the diet and $60 \%$ performing regular physical activity. There was also an increase in medical consultations with the family doctor by $52 \%$. As final conside-rations, it can be said that there was a decrease in cases of seeking care for hypertensive crises, demonstrating that continuous medical monitoring and health policy actions in a multidisciplinary context can be decisive to ensure a better quality of life for users.

Keywords: Community health; Chronic diseases; Health education.

*Autor correspondente: Alberto Furini Vaz, Médico, Farmacêutico, Rua Presidente Dutra 135, Antonio Joao, MS, 67-99138-3590, albertofurinivaz@gmail.com 


\section{INTRODUÇÃO}

A Hipertensão Arterial Sistêmica (HAS) ou pressão alta é conceituada como doença crônica não transmissível caracterizada por um desequilíbrio entre pressão sistólica e diastólica. É diagnosticada a partir de valores de máxima e mínima iguais ou maiores que os 140/90 mmHg (MALACHIAS et al., 2016).

Por estar em desequilíbrio a HAS provoca no indivíduo a necessidade de um esforço maior ao músculo cardíaco. Com o tempo o indivíduo começa a sentir cansaço, indisposição, transtornos do sono, tontura, dores de cabeça, entre outros sintomas. Pode se não tratada provocar acidente vascular cerebral, infarto, aneurisma arterial e insuficiência renal e cardíaca, entre outras complicações (BRASIL, 2013).

Acredita-se que na atualidade a HAS atinja cerca de 1,13 bilhão de pessoas no mundo, e não é mais considerada como doença típica de países ricos. No Brasil acredita-se que haja entre os indivíduos maiores de 40 anos $30 \%$ de prevalência. Antes uma doença de idosos, hoje atinge inclusive indivíduos jovens com 20 a 30 anos. É muito comum homens apresentarem o problema (CAMPOS et al., 2019).

Dentre as principais causas da hipertensão aponta-se o tabagismo, drogadição, consumo de bebidas alcoólicas, obesidade, estresse, sedentarismo, elevado consumo de sal, níveis altos de colesterol, falta de atividade física, além de fatores genéticos, cor, biótipo dentre outros (MALTA et al., 2018; CAMPOS et al., 2019).

É muito comum usuários hipertensos apresentarem outras comorbidades, como dislipidemias e diabetes, o que agrava ainda mais a possibilidade de surgimento de consequências. Quanto aos sintomas depende muito do usuário. Em alguns indivíduos podem surgir dores no peito, dor de cabeça, tonturas, zumbido no ouvido, fraqueza, visão embaçada e sangramento nasal. Contudo não é regra. Alguns indivíduos são hipertensos e não apresentam sintomas evidentes (MALTA et al., 2018).

Um dos principais objetivos da Estratégias de Saúde da Família (ESF) é o controle das doenças crônicas não transmissíveis. Cito uma alternativa proposta pelo Governo Federal que é a implementação do Programa Mais Médicos Para ao Brasil, ao oferecer aos Municípios Brasileiros um profissional para a Atenção Primária à Saúde (APS) que possa dentre as muitas ações desenvolver um projeto de intervenção com relação a um problema diagnosticado na comunidade, que neste caso foi a hipertensão.

$O$ tratamento (controle) da HAS é realizado com mudanças de hábitos de vida, e intervenção medicamentosa com anti-hipertensivos e diuréticos. Com relação as mudanças de hábitos de vida estão relacionadas a melhoria da alimentação, com aumento do consumo de verduras, frutas, carnes brancas e magras, baixo consumo de óleos (salvo o azeite de oliva), baixo consumo de açúcar e sódio, atividade física regular, equilíbrio emocional, qualidade de sono, entre outros. $O$ tratamento medicamentoso depende muito do usuário e as reações que terão as medicações em seu organismo (DANTAS; RONCALLI, 2019).

Nesse sentido apresenta-se a realidade da ESF rural localizada em Ponta Porã, estado do Mato Grosso do Sul. Atualmente tem cerca de 618 usuários cadastrados e destes, 138 apresentam diagnóstico de HAS. São em sua grande maioria idosos e indivíduos do sexo masculino.

O objetivo foi a realização de ações de educação em saúde na prevenção e combate a hipertensão com este público, bem como evidenciar a importância da atuação do médico da família em comunidades rurais distantes e com dificuldade de acessibilidade, podendo servir de modelo para a organização de programas de intervenção e prevenção em outras comunidades com problemas similares.

\section{MATERIAL E MÉTODOS}

Trata-se de um trabalho realizado no formato de Projeto de Intervenção (PI), desenvolvido com os usuários HAS cadastrados em uma ESF rural que abrange assentamentos, fazendas e moradores locais localizados em Ponta Porã/MS.

Os dados foram extraídos de fontes pri- 
márias e das atividades de educação em saúde realizadas nos anos de 2020 e 2021 , com os usuários que apresentaram interesse em participar dos encontros e ações.

A primeira etapa do projeto foi a capacitação da equipe de saúde para o manejo da prevenção e o combate a HAS, utilizando-se do Caderno de Atenção Básica $n^{\circ} 37$ do Ministério da Saúde como elemento norteador. Em seguida, realizou-se a identificação de usuários a participarem das intervenções e o convite foi realizado pelos agentes comunitárias de saúde (ACS) e equipe de saúde durante as visitas domiciliares. Inicialmente formou-se um grupo de 20 participantes que receberam instruções sobre a HAS e suas consequências Acidente Vascular Cerebral (AVC), Infarto Agudo do Miocárdio (IAM), entre outros, bem como o acompanhamento clínico do médico, incluindo visita domiciliar.

A terceira e última etapa foi a sensibilização dos usuários quanto às mudanças de hábitos de vida e adesão ao tratamento. Foram realizadas orientações individuais e coletivas por meio de atividades educativas, telemedicina, consulta com cardiologista e medicina remota com os usuários, utilizando-se de celulares e aplicativos específicos. Estas ações foram realizadas em virtude da necessidade de obedecer às medidas de isolamento referentes a ações relacionadas a prevenção ao covid-19.

Outros temas também foram abordados como: alimentação saudável, sedentarismo, ingestão de sódio, equilíbrio emocional, qualidade do sono, adesão ao tratamento medicamentoso e consequências do descontrole dos valores pressóricos.

\section{RESULTADOS E DISCUSSÃO}

Antes de descrever o relatório sobre as ações realizadas é oportuno destacar que a área onde se desenvolveu o presente PI é rural, de difícil acesso, havendo assentamentos distantes e até uma aldeia indígena na região, onde provisoriamente realiza-se o atendimento quando a falta de médico do Distrito Sanitário Especial de Saúde Indígena (DSEI).
Há usuários residentes em fazendas e assentamentos que se encontram a 30 quilômetros de distância da Unidade de Saúde da Família (USF). A dificuldade em se deslocar até a cidade para acompanhamento médico resulta no agravamento da patologia.

Contemporaneamente, as necessidades de saúde da população rural são mais complexas se comparadas às urbanas (LIMA et al., 2019). Por vivenciarem, cotidianamente, maiores desafios e obstáculos para acessarem os serviços de saúde torna-se imprescindível direcionar ações e iniciativas que reconheçam as especificidades desses territórios objetivando a melhoria dos indicadores e da qualidade de vida, aproximando essas populações da integralidade do cuidado (PONTES et al., 2018).

O início do projeto ocorreu com a capacitação da equipe de saúde para o manejo da prevenção e o combate a HAS, utilizando as recomendações do Caderno de Atenção Básica $n^{\circ}$ 37. A capacitação, presidida pelo médico da unidade com auxílio da enfermeira, ocorreu nas dependências da ESF com a presença e o envolvimento de todos os membros da equipe de saúde (1 médico, 4 ACS, 1 enfermeiro, 2 auxiliares de enfermagem, 1 recepcionista, 1 administrativo e 1 odontólogo). Foram definidas responsabilidades de cada membro nas ações, seus objetivos, a exposição e construção dos temas abordados com os usuários, bem como as dinâmicas de realização das ações, datas, cronogramas, controladores e resultados esperados.

Segundo Peduzzi e Agreli (2018) o trabalho em equipe é um processo dinâmico no qual os profissionais se conhecem e aprendem a trabalhar juntos para reconhecer o trabalho, conhecimentos e papéis de cada profissão.

Não houve necessidade de uma segunda reunião, uma vez que a primeira foi bastante esclarecedora. Outrossim, alguns pequenos ajustes foram realizados em reuniões semanais de rotina da equipe.

Após a capacitação os ACS e a equipe de saúde começaram a convidar os usuários para participar das atividades educativas. As ferramentas utilizadas foram a roda de conversa no 
espaço físico da própria unidade de saúde que possui uma área coberta, cadeiras e com plenas condições de abrigar os usuários.

Para Peduzzi e Agreli (2018) na atividade educativa a comunicação se caracteriza especialmente pela comunicação efetiva interprofissional e com usuários e população na construção de parcerias.

A roda de conversa é utilizada como método para envolver a participação popular no planejamento de ações em saúde. Para desenvolver estratégias educativas condizentes com as práticas culturais de determinada comunidade é preciso tonar os usuários protagonistas do processo de aprendizagem (MACHADO et al., 2015).

A primeira reunião apresentou uma adesão bastante baixa (11 participantes) dos usuários, contudo durante a semana, nos atendimentos cotidianos e na renovação de receitas os usuários foram incentivados a participarem das atividades educativas, fazendo com que houvesse mais pessoas. Assim, no segundo encontro compareceram 17 pessoas, sendo os mesmos 11 da primeira reunião somados a outros 6 novos participantes.

Nesta reunião percebeu-se que os usuários estavam um pouco dispersos, e sem entender a finalidade das atividades visto que tal prática não era realizada fazia muito tempo. No entanto, após os esclarecimentos, fundamental para a compreensão da proposta, os usuários apresentaram boa interação com a equipe de saúde.

Nessa logística foram realizados ainda dois outros encontros presenciais, com intervalo de média de 30 dias entre eles e sempre com mais pessoas participando.

Os temas abordados foram a definição da hipertensão e os males que a mesma causa quando descontrolada, como a possibilidade de ocorrências de IAM e AVC, as consequências do sedentarismo, a importância da dieta, os malefícios do tabaco e alcoolismo, do excesso de peso, imunização, rotina de atendimento da unidade, como por exemplo, a forma de agendamento dos exames.

Em todos os encontros, buscou-se a autoconfiança e independência dos usuários tor- nando-os capazes de mudar o estilo de vida e influenciar o meio em que vivem. Desse modo, a educação em saúde foi considerada como um processo de ensino aprendizagem, onde as particularidades da população rural foram valorizadas (CARVALHO \& GASTALD, 2008).

As atividades educativas permitiram que as comunidades rurais participassem ativamente desde o planejamento das ações até a tomada de decisões sobre as necessidades de saúde e utilização dos recursos do território. Foram aproveitados os espaços de visitas domiciliares para reforçar as informações relativas à educação em saúde, verificar o uso adequado das medicações, adesão à dieta e atividades físicas, além do controle da ingestão de sódio.

As ações presenciais foram realizadas até o início da Pandemia do Covid-19, sendo que após foram necessários reunir esforços e criatividade da equipe de saúde na reorganização das atividades educativas e dos atendimentos a fim de dar continuidade ao seguimento dos usuários.

Assim, o desenvolvimento da proposta sofreu alterações por meio da implantação da telemedicina com os usuários do HIPERDIA, com o apoio da secretaria municipal de saúde que disponibilizou um aparelho telefônico móvel com acesso a internet. Montou-se um grupo de WhatsApp com os usuários hipertensos e deu-se continuidade às atividades educativas de modo virtual.

Dentre as ações relacionadas a telemedicina, de relevância nesse trabalho, está o desenvolvimento de um "Hiperdia Virtual" além de gravação e publicação semanal de vídeos curtos (cerca de 10/15 minutos de duração) no grupo do Hiperdia. Além disso algumas consultas foram realizadas utilizando videochamada, entre outras ações.

O trabalho virtual foi possível, pois a maioria dos usuários tinham acesso à internet e os que não tinham, a estratégia alternativa foi cadastrar o telefone de algum parente ou amigo próximo e inseri-lo no grupo do hiperdia. Assim, nos fins de semana os hipertensos podiam ter cesso ao vídeo na ocasião das visitas dessas pessoas. 
A fim de incentivar a participação dos usuários, foram realizadas ações com pequenos desafios e possibilidade de premiação. Por exemplo, nas orientações relacionadas a alimentação correta, foi solicitado aos usuários que postassem fotos de pratos saudáveis e aquelas que melhor representassem o tema abordado, participava da realização de sorteios (grupo de whatsapp) e prêmio ao vencedor foi um termômetro digital.

A telemedicina e atenção remota foram utilizadas frente a realidade existente trazida pela pandemia. Os usuários hipertensos e diabéticos estão enquadrados no chamado de "grupo de risco", ou seja, pessoas que por sua condição de doença crônica apresentam uma maior possibilidade de evolução da doença (no caso da covid) que os demais; justamente por isso demandam um cuidado maior, tanto da doença, quanto da prevenção, por isso a importância da telemedicina e atenção remota nestes casos (MELO et al., 2020).

Estas ações têm sido uma alternativa para que o controle da doença crônica seja continuado. Isso porque as medidas de isolamento trouxeram uma perca de foco as demais doenças, incluindo a HAS. A pandemia causada pelo novo coronavírus fez com que muitos usuários evitassem as ESF e o controle da PA. Nesse sentido as ações foram desenvolvidas no intuito de dar continuidade ao plano de ações evitando o mínimo de prejuízo aos usuários assistidos.

A estratégia do WhatsApp teve boa aceitação pela comunidade que participou ativamente; permitiu que os usuários ficassem mais calmos, seguros e tranquilos, pois sabiam que teriam acesso ao médico mesmo com as dificuldades relativas ao isolamento social.

Quanto à organização da agenda médica, havia um período por semana em que o grupo era visitado e as perguntas eram atenciosamente respondidas pelo mesmo.

Com relação aos dados coletados destes usuários houve ótimos resultados. Ao todo são 138 hipertensos cadastrados o que representa $28,45 \%$ da população de 14 a 95 anos de idade. Do total de hipertensos, 24 possuem diabetes como patologia associada.

Quando se iniciou os trabalhos na ESF abrangendo as quatro regiões adscritas, deparou-se com muitos casos de crises hipertensivas. Com o desenvolvimento das ações do PI $85 \%$ dos usuários conseguiram melhorar os índices pressóricos e mantê-los dentro do padrão de normalidade.

A prevenção e o manejo da HAS são ações programáticas prioritárias na ESF por serem consideradas condições sensíveis a esse nível de atenção, havendo evidência acumulada de que o bom manejo desses problemas, na APS evita hospitalizações e mortes decorrentes de suas complicações (FONTBONNE et al., 2018).

Em uma entrevista coletiva no último encontro, $80 \%$ das pessoas afirmaram estar consumindo menos sódio na dieta; $60 \%$ afirmaram estar fazendo atividade física regular, algumas caminhadas, e alguns na academia pública, foi considerado aqueles que também são braçais como atividade física regular.

Em um estudo epidemiológico transversal conduzido em 2010 em uma amostra representativa de usuários hipertensos e diabéticos tipo 2, com mais de 20 anos de idade, cadastrados na Estratégia Saúde de Família do Estado de Pernambuco, baseado no estudo SERVIDIAH (Avaliação de Serviços de atenção à saúde para Diabéticos e Hipertensos no âmbito da Estratégia Saúde da Família), observou-se que menos da metade dos hipertensos tinha pressão arterial controlada e que, apesar de cerca de $75 \%$ dos entrevistados apresentarem excesso de peso, somente $16 \%$ dos hipertensos disseram seguir uma dieta para perder peso, enquanto $70 \%$ afirmaram não praticar nenhuma atividade física (FONTBONNE et al., 2018).

Quando a promoção da atividade física pelo serviço é feita de maneira engajada e repetida, ela surte efeitos em usuários com condições crônicas (NIED \& FRANKLIN, 2012). Assim, vale a pena continuar o trabalho educativo, pois certamente a médio e longo prazo os resultados poderão ser melhores.

Segundo o Ministério da Saúde (2013), as medidas não farmacológicas mais efetivas para 
controlar a HAS e diminuir os riscos cardiovasculares são redução de peso, adoção de hábitos de vida e alimentares saudáveis. Dessa forma, a atividade física, a dieta hipossódica e hipolipídica associados, permitem a avaliação nutricional da pessoa e oferecem subsídios para a prevenção e controle da HAS (BRASIL, 2013).

Antes da implantação do PI, os médicos especialistas não atendiam na localidade e os usuários se deslocavam até a cidade, contudo os usuários tinham dificuldade até mesmo pra chegar à ESF pois muitos dependem de carona em carro privado, do ônibus escolar, de carroça, visto que a maioria não possui transporte próprio.

Nesse âmbito, a chegada do médico de família na unidade a partir de abril de 2019, propiciou um aumento significativo no número de consultas médicas na ESF de $52 \%$ para o primeiro ano de trabalho, passando de 6.485 para 9.836 atendimentos (os dados foram tirados do programa GSEA, ferramenta de informática utilizada para gerenciamento de sistema de gestão em saúde, educação e assistência social do município).

Além do aumento das consultas, a população também teve um ganho ponderal na qualidade da assistência prestada e pelas conquistas das novas pactuações que trouxeram o médico cardiologista para o atendimento mensal, auxiliando no tratamento medicamentoso e realizações de eletrocardiograma aos usuários referendados. A equipe de saúde também ajudou muito, participando das ações, estruturando um modelo aplicado a realidade de ESF Rural.

A Sociedade Brasileira de Medicina de Família e Comunidade (SBMFC), alerta sobre os riscos que a população rural corre, em tempos de pandemia. Sabe-se que praticamente metade da população mundial reside em áreas rurais, sendo que, menos de $1 / 4$ dos profissionais de saúde trabalham nesses locais. Contudo, se faz sumamente importante e necessário se adequar frente as adversidades para flexibilizar a atenção médica em usuários de alto risco com patologias crônicas.

A ênfase do projeto não está somente na captação e seguimento de usuários com patologias crônicas, visando a estratificação de risco, pois estes dados são exigidos para todas as Estratégias de Saúde da Família (ESF) como planiIha controle do sistema e SUS e indicadores do Previne Brasil (sistema de alimentação de dados exigido e interligado com Ministério da Saúde).

\section{CONCLUSÃO}

As doenças crônicas são de fato um problema de saúde pública. Atualmente diabetes e hipertensão representam uma grande quantidade dos atendimentos da Atenção Primária. Os resultados correspondentes aos objetivos foram alcançados sendo que o PI atendeu as expectativas do "Programa Mais Médicos para o Brasil", à medida que as ações foram realizadas em conjunto, entre ações do Governo Federal e Prefeitura Municipal de Ponta Porã. Os dois trabalhando em perfeita simetria trouxeram resultados sólidos aos usuários da ESF rural, beneficiando-os.

Não houve limitações além das já relatadas no próprio decorrer da proposta, principalmente relacionadas a pandemia e a limitação do contato. Como resultados percebeu-se uma diminuição significativa das crises hipertensivas e queixas de alteração da pressão arterial na unidade.

Como plano de continuidade o Programa HIPERDIA, continua funcionando uma vez por semana, com consultas médicas para ajustes dos medicamentos, orientação sobre alimentação, atividade física dentre outras ações.

Em virtude de a continuidade da proposta ter se dado em período de afastamento social, foram adaptadas medidas de telemedicina, para que se mantivessem os encontros de Hiperdia, antes realizados na Unidade Básica. $\mathrm{O}$ seguimento está ocorrendo por meio de encontros e mensagens virtuais (whatsapp), por meio de telefone celular fornecido pela prefeitura municipal para agendamentos, avisos e realização de grupos para repasse de informações, um recurso buscado para minimizar os efeitos relacionados ao afastamento social em usuários com patologias crônicas. 
As medidas que estão sendo adotadas no ESF rural de Ponta Porã podem servir de modelo a outras unidades rurais interessadas em implementar o projeto em tempos de pandemia. O curso de especialização oferecido pela FIOCRUZ foi determinante para a realização do projeto, através dele foi possível aplicar o conhecimento obtido no decorrer do curso, juntando a pratica com a teoria, trazendo inclusive grandes ganhos a comunidade.

\section{REFERÊNCIAS}

BRASIL. Ministério da Saúde. Secretaria de Atenção à Saúde. Departamento de Atenção Básica. Estratégias para o cuidado da pessoa com doença crônica: hipertensão arterial sistêmica / Ministério da Saúde, Secretaria de Atenção à Saúde, Departamento de Atenção Básica. Brasília: Ministério da Saúde, 2013. 128 p.: il. (Cadernos de Atenção Básica, n. 37)

CAMPOS, K.F.C; et al., Educação permanente em saúde e modelo assistencial: correlações no cotidiano do serviço na Atenção Primária a Saúde. APS EM REVISTA, v. 1, n. 2, p. 132-140, jul., 2019.

CARVALHO, S.R.; GASTALD, D. Promoção à saúde e emponderamento: uma reflexão a partir das perspectivas crítico-social pós-estruturalista. Ciênc. Saúde Coletiva, v.13, n.2, p 2029-2040, 2008. Disponível em:<http:// www.scielo.br/scielo.php?script=s ci_arttext\&pi$\mathrm{d}=$ S1413- 81232008000900007> Acesso em 28 junho de 2020.

DANTAS, R.C.D.O; RONCALLI, A.G. Protocolo para indivíduos hipertensos assistidos na Atenção Básica em Saúde. Ciência e Saúde Coletiva, v. 24, n. 1, p. 295-306, janeiro, 2019. Disponível em<http://www. scielo.br/scielo.php?script=sci_arttext\&pi$\mathrm{d}=$ S1413-81232019000100295>.Acesso em 07 de fevereiro de 2020.

FONTBONNE, A.; et al. Relações entre os atributos de qualidade de atenção aos usuários hipertensos e diabéticos na Estratégia Saúde da Família e o controle dos fatores prognósticos de complicações. Cad. saúde colet., v. 26 , n. 4, p. 418-424, dezembro 2018. Disponível em: <http:// www.scielo.br/scielo.php?script=sci_arttext\&pi$\mathrm{d}=\mathrm{S} 1414=462-2018000400418 X \&$ Ing=en\&nrmiso>. Acesso em 28 Junho de 2020.

LIMA, A.R.A.; et al. Necessidades de saúde da população rural: como os profissionais de saúde podem contribuir? Saúde debate, v. 43, n. 122, p. 755-764, 2019. Disponível em:<http:// www.scielo.br/scielo.php?script=sci_arttext\&pi$d=$ S0103-11042019000300755\&Ing=en\&nrm=iso>. Acesso em 28 junho 2020.

MACHADO, T.M.G.; CARVALHO, P.I.N.; BRANDÃO, A.D.S.M.; VILARINHO, M.L.C.M.; A roda de conversa como ferramenta de planejamento de ações: relato de experiência. Rev. G\&S [Internet]., v.1, n. 1, pag. 751-761. Disponível em:<https://periodicos.unb.br/index.php/ rgs/article/view/2707>. Acesso em 28 junho 2020.

MALACHIAS, M.V.B. et al. $7^{\text {a }}$ Diretriz Brasileira de Hipertensão Arterial. Arquivos Brasileiros de Cardiologia, v. 107, n. 3, Supl.3, p. 1-83, 2016. Disponível em:<http://publicacoes. cardiol.br/2014/diretrizes/2016/05_HIPERTENSAO_ARTERIAL.pdf>. Acesso em 28 junho 2020.

MALTA, Deborah Carvalho et al., Prevalência da hipertensão arterial segundo diferentes critérios diagnósticos. Pesquisa Nacional de Saúde. Rev. bras. epidemiol., v. 21, Supl. 1, 2018 . Disponível em:<http://www. scielo.br/scielo.php?script=sci_arttext\&pid=S1415-790X2018000200419>Acesso em 07 de fevereiro de 2020.

MELO, D.O.D.; et al. COVID-19 e doença hipertensiva no Brasil: possibilidade de uma tempestade perfeita. Rev. Bras. Epidemiol., v. 23, 2020.Disponível em:<https://www.scielosp. 
org/pdf/rbepid/2020.v23/e200062/pt>Acesso em 22 de maio de 2021.

NIED, R.J.; FRANKLIN, B. Promoting and prescribing exercise for the elderly. Am Fam Physician, v.65, n. 3, p. 419-26, 2012.

PEDUZZI, M.; AGRELI, H.F.. Trabalho em equipe e prática colaborativa na Atenção Primária à Saúde. Interface (Botucatu), v. 22, supl. 2, p. 1525-1534, 2018. Disponível em: <http:// www.scielo.br/scielo.php?script=sci_arttext\&pi$\mathrm{d}=$ S1414=32832018000601525-\&Ing=en\&nrmiso>. Acesso em 28 Junho de 2020.

PONTES, A.G.V.; RIGOTTO, R.M.; SILVA, J.V. Necessidades de saúde de camponeses em conflito ambiental frente à instalação de Perímetros Irrigados. Ciênc. Saúde Colet. [internet], v. 23, n. 5, p. 1375-1386, 2018. Disponível em:<http://www.scielo.br/pdf/csc/v23n5/ 1413-8123-csc-23-05-1375.pdf.> Acesso em 07 de fevereiro de 2020. 